\title{
eJRIEPS
}

Ejournal de la recherche sur l'intervention en éducation physique et sport

$30 \mid 2013$

Varia

\section{Activité technique en contexte sportif : point de vue en technologie des pratiques physiques sportives et artistiques}

Daniel Bouthier

\section{(2) OpenEdition}

\section{Édition électronique}

URL : http://journals.openedition.org/ejrieps/2485

DOI : $10.4000 /$ ejrieps.2485

ISSN : 2105-0821

Éditeur

ELLIADD

Référence électronique

Daniel Bouthier, «Activité technique en contexte sportif : point de vue en technologie des pratiques physiques sportives et artistiques », eJRIEPS [En ligne], 30 | 2013, mis en ligne le 01 juillet 2013, consulté le 07 octobre 2019. URL : http://journals.openedition.org/ejrieps/2485 ; DOI : 10.4000/ ejrieps. 2485

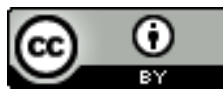

La revue eJRIEPS est mise à disposition selon les termes de la Creative Commons Attribution 4.0 International License. 
eJRIEPS 30 juillet 2013

Activité technique en contexte sportif : point de vue en technologie des pratiques physiques sportives et artistiques

Daniel Bouthier

Laboratoire «Cultures, Education, Sociétés », équipe «Vie Sportive » (EA4140)

Université de Bordeaux

\section{Introduction : pratique et activité, sortir de la confusion.}

Nous distinguerons ici des pratiques socio-historiquement élaborées et des activités individuellement déployées. Ce qui ne signifie pas pour autant indépendance entre ces deux pôles. II semble en effet possible d'avancer que les pratiques participent d'un contexte, d'une structure d'accueil et de déploiement pour les activités. Réciproquement les activités humaines paraissent largement engagées dans l'émergence et l'évolution des pratiques.

\section{Des activités techniques, c'est-à-dire.}

L'activité humaine est explicitée, notamment par Léontiev (1976) puis Clot (1995), à trois niveaux.

- Un premier niveau englobant renvoie aux motifs personnels d'agir eux-mêmes en relation dialectique avec les significations sociales des pratiques.

- Un deuxième niveau qui matérialise le précédent, se produit dans l'action orientée et organisée par rapport à des buts conscients retenus dans la tâche effective.

- un troisième niveau qui réalise le niveau précédent, met en jeu des opérations tacites ou explicites, constituant les moyens d'atteindre le but envisagé.

Parmi les différents types d'activités humaines possibles, certaines d'entre elles sont déployées à des fins de transformations intentionnelles, utilitaires ou symboliques. Nous parlerons alors "d'activités techniques " qui se manifestent, à travers des situations de production par la mobilisation d'objets techniques (outils, instruments ou artefacts) quels que soient leur nature, leurs caractéristiques, leur champ d'application (Simondon 1935), et par la mise en jeu d'une certaine technicité. Ces artefacts peuvent être de différentes natures: cognitifs (Norman 1993), matériels (Rabardel 1995), mais aussi corporels (Mauss 1936). La contribution des artefacts à la fonctionnalité et au développement de l'activité technique a été approfondie à travers sa fonction instrumentale. II faut en effet distinguer l'artefact en tant qu'outil avec ses propriétés, créé et mis à disposition par 


\section{eJRIEPS 30 juillet 2013}

d'autres hommes et, l'usage et l'appropriation de cet artefact devenant un instrument de l'activité technique d'un individu singulier. L'efficience (efficacité et économie) dans la mise en œuvre des artefacts repose sur l'acquisition de compétences spécifiques (connaissances et expériences) ou technicité qui met en jeu une rationalité (intelligence pratique), des artefacts (procédures et outils originaux), et une distribution des rôles (socio-techniques), spécifiques (Combarnous 1984). Cette technicité, du point de vue de son acquisition et de son enseignement, se déploie selon Martinand (1994) à travers quatre registres interdépendants qui rendent compte à la fois de la dimension sociale et de la dimension personnelle de l'activité technique.

\section{Des activités en contexte, c'est-à-dire.}

Nous avons évoqué en introduction la distinction pratiques/activités en indiquant que les pratiques constituaient un des éléments contextuels des activités. Cela demande un approfondissement. La notion de contexte renvoie à des pratiques sociales historiquement et culturellement produites, accumulées et transmises, qui sont en grande partie immatérielles. Elle est à distinguer de l'environnement qui lui est physique et donc plus perceptible, qu'il soit matériel ou humain. Les activités techniques se déroulent ainsi dans le cadre de situations de pratiques instrumentées qui mettent en présence une ou des tâches prescrites avec des résultats attendus, dans un dispositif organisant un système d'outils. Ces outils ne viennent pas de nulle part, ils sont un produit social porteur de conceptions et de façons de faire cristallisées dans les pratiques. "En attribuant à un outil des finalités et des fonctionnalités conformes à celles prévues par son concepteur, l'utilisateur " récupère » en quelque sorte l'intelligence «cristallisée » dans l'artefact » (Vérillon, 2005, p. 315). "Enfin, les paramètres de la tâche dans laquelle s'inscrit l'activité instrumentée peuvent considérablement conditionner les interactions. Ainsi l'activité instrumentée développée présentera des caractéristiques différentes en situation d'apprentissage, en situation de compétition, en situation d'urgence ou en mode de fonctionnement dégradé, ou encore, selon que le projet est à dominance épistémique ou pragmatique, ou selon que l'action considérée est individuelle ou distribuée sur un collectif » (Vérillon 2005, p. 313). Considérant le sport comme une composante de la culture et une activité productive complexe, Vérillon (2005, p. 305) rappelle que «la performance est une réalisation qui implique, au-delà de l'athlète individuel ou collectif, l'existence d'un encadrement, d'une organisation, d'infrastructures qui contribuent de manière décisive à sa production ». 


\section{eJRIEPS 30 juillet 2013}

\section{Perspectives technologiques d'analyse de l'activité en contexte sportif.}

En troisième lieu nous développons quelques pistes théoriques et méthodologiques pour une analyse technologique de l'activité en contexte sportif. Si par technologie nous entendons une science humaine des techniques (Haudricourt 1987), nous considérons que la technologie des pratiques physiques sportives et artistique porte sur deux dimensions :

- d'une part l'analyse des techniques corporelles complexes mises en jeu dans les pratiques sportives,

- d'autre part l'analyse des techniques d'intervention mises en œuvre par l'intervenant éducatif (enseignant, moniteur, entraîneur) pour optimiser la transmission des techniques sportives (Bouthier, 1993 ; Bouthier \& Durey, 1994).

Chacune d'entre elles suppose un plan central d'étude portant sur les techniques en usage et les essais techniques dans les situations de pratique instrumentées, constituées du couple tâche prescrite $\Leftrightarrow$ activité effective en environnement naturel. Pour autant cela ne suffit pas à épuiser la connaissance, la compréhension et la capacité d'intervenir pour en transformer la performance produite. Deux autres plans d'analyse s'avèrent indispensable :

- un plan plus macroscopique éclairant les phénomènes de continuité et rupture des lignées techniques, et des axes potentiels de développement, recontextualisés notamment à travers les problèmes qu'ils visent à résoudre, les connaissances à disposition (scientifiques et professionnelles), les procédures en usage et les expériences possibles, ainsi que les organisations qui les accueillent.

- un plan plus microscopique qui nécessite d'expliciter les rapports subjectifs des sujets engagés dans les activités techniques et confrontés aux objets de la culture technique. Quelles intentions initiales, quelles focalisations, quels horizons d'attente, ces activités répondent du point de vue du sujet agissant.

Ces recherches technologiques poursuivent une finalité de transformation du système technique par l'introduction de nouveaux artefacts (corporels, matériels et/ou cognitifs). Elles supposent non seulement l'étude du processus et des procédures prévus et effectifs, mobilisés dans la conception, la mise en œuvre et l'évaluation des essais techniques innovants, mais aussi de dégager les nouveaux savoirs technologiques ainsi mis à jour. 


\section{eJRIEPS 30 juillet 2013}

Schéma 1. Les pratiques sportives comme contexte et inducteur d'activités techniques.

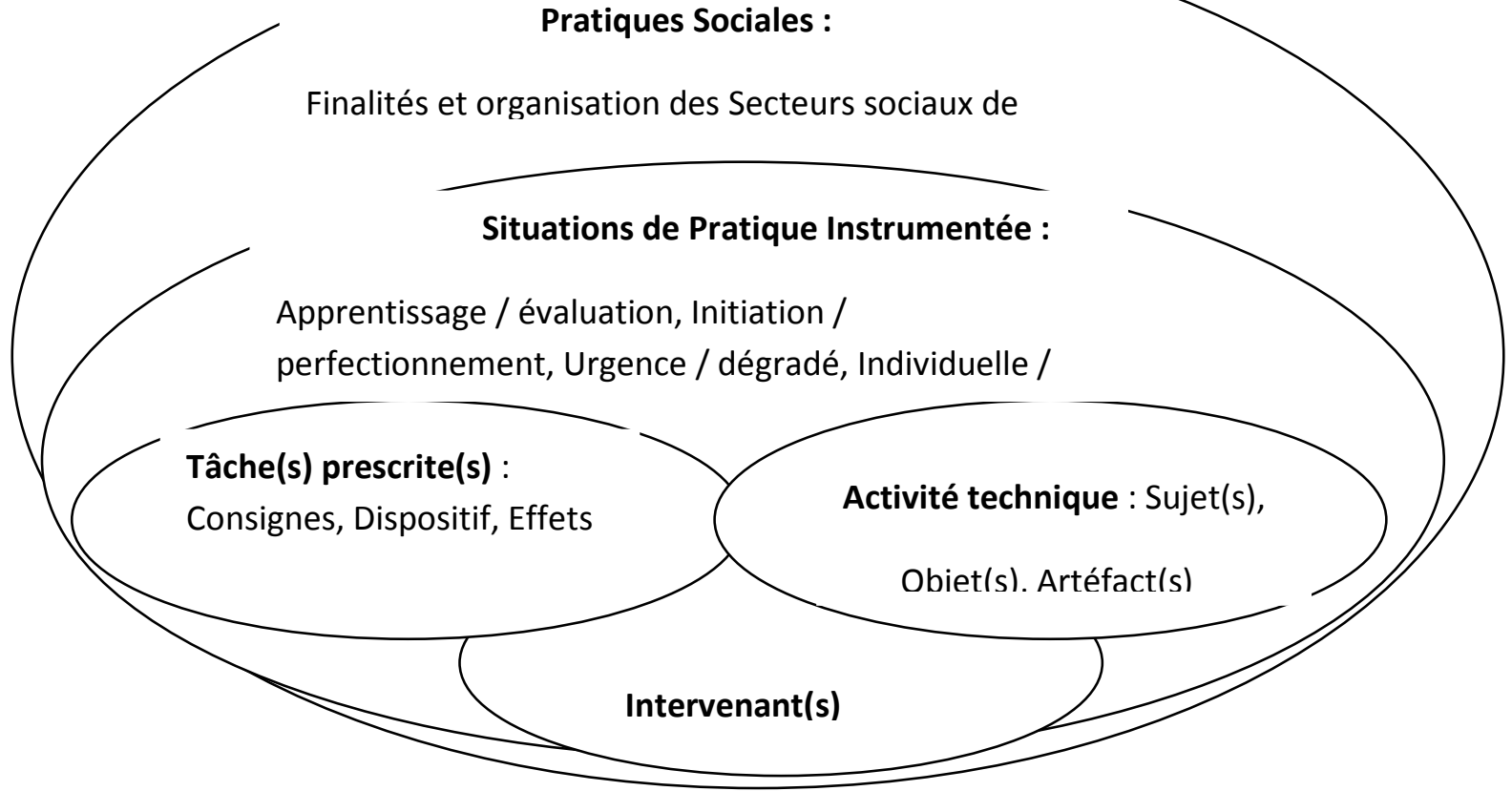

\section{Conclusion}

L'analyse des activités techniques en contexte sportif permet d'explorer les capacités humaines de développement et de mobilisation des pouvoirs d'agir corporellement sur soi, sur autrui, et sur l'environnement physique. Elle constitue une voie spécifique d'approche de l'activité créatrice engagée dans l'intelligence pratique de transformation du monde objectif, tout en reposant sur les rapports subjectifs singuliers entretenus par chacun avec les artefacts, le système et la culture technique. Bien évidemment, en accord avec Vérillon (2005) l'activité technique déployée par chacun est impactée par les caractéristiques des situations sportives, selon qu'il s'agit de situations d'initiation, de perfectionnement ou d'excellence, d'entraînement ou de compétition, d'urgence ou de confort, en pleine potentialité ou diminuées, individuelles ou collectives, ...

\section{Bibliographie}

Bouthier D. (1993). L'approche technologique en STAPSQ : représentations et actions en didactique des APS. HDR, Université Paris-Sud, document non publié, 117 p.

Bouthier D., Durey A. (1994). Technologie des APS. Impulsions, 1, 95-124.

Clot Y. (1995). La compétence en cours d'activité. Education Permanente, 123, 115-123. 


\section{eJRIEPS 30 juillet 2013}

Combarnous M. (1984). Les techniques et la technicité. Messidor/Éditions sociales.

Haudricourt A. G. (1987). La technologie science humaine. Paris : Maison des Sciences de l'Homme.

Leontiev A. (1976). Le développement du psychisme. Paris : Editions Sociales.

Leontiev A. (1984). Activité, conscience, personnalité. Moscou : Editions du progrès.

Martinand J.L. (1994). La didactique des sciences et de la technologie et la formation des enseignants. ASTER, 19, 61-75.

Norman D. A. (1993). Les artefacts cognitifs. Raisons Pratiques, 4, 15-34.

Rabardel P. (1995). L'homme et les technologies. Approche cognitive des instruments contemporains. Paris : Armand Colin.

Simondon G. (1935). Du mode d'existence des objets techniques. Paris: Aubier Montaigne.

Vérillon P. (2005). Processus productifs et constructifs dans les activités physiques et sportives : la place de l'instrument. Impulsions, 4, 305-325. 